\title{
Refeeding Hospitalized Patients with Eating Disorders: Does Gender Matter?
}

\author{
Sarah E Strandjord ${ }^{\mathrm{a}}$, Erin H Sieke ${ }^{\mathrm{a}}$, Miranda Richmond ${ }^{\mathrm{b}}$, Ellen S Rome ${ }^{\mathrm{c} *}$ \\ ${ }^{a}$ Cleveland Clinic Lerner College of Medicine, Cleveland, $\mathrm{OH}$ \\ ${ }^{b}$ Cleveland Clinic Office of Civic Education Initiatives, Beachwood, $\mathrm{OH}$ \\ ${ }^{c}$ Cleveland Clinic Children's Hospital, Department of General Pediatrics, Cleveland, $\mathrm{OH}$
}

Received: September 16, 2016; Accepted: October 30, 2016; Published: December 29, 2016

*Corresponding author: Ellen S Rome, Cleveland Clinic Children's Hospital, Department of General Pediatrics, 9500 Euclid Avenue, A120, Cleveland, OH 44195, Tel: 216.444.3566; Fax: 216-445-3523; E-mail: romee@ccf.org

\begin{abstract}
Purpose: Eating disorder research typically focuses on females with few studies reporting gender comparisons. The present study compares male and female patients hospitalized for refeeding, including differences at presentation, during hospitalization, and one year after admission.
\end{abstract}

Methods: We conducted a chart review of eating disorder patients hospitalized between 2008 and 2014 for acute medical stabilization at an academic medical center. Data, including characteristics at presentation, responses to inpatient treatment, and outcomes one year after admission, were recorded for each patient. Male and female patients were compared using the Kruskal-Wallis test for continuous variables and the Chi-square test for categorical variables.

Results: At presentation, males $(\mathrm{n}=38$ ) had shorter durations of illness but greater weight loss than females $(n=302)$. Males exhibited less purging behaviors but more illicit drug use than females, with no difference in psychiatric comorbidity. During hospitalization, despite similar dietary interventions and rates of weight gain, length of stay was significantly longer for males than for females ( 6.5 vs. 5 days, $p=$ 0.007 ). One year after admission, less than one-quarter of male and female patients required readmission $(18 \%$ vs. $21 \%, p=0.74)$ and around half of patients met criteria for remission $(64 \%$ vs. $49 \%, p=$ $0.19)$.

Conclusions: Differences between males and females with eating disorders may affect their treatment needs during and after hospitalization. Similar outcomes across gender one year after hospitalization highlight both the success and the continued need for improvement in eating disorder treatment regardless of gender.

Keywords: eating disorder, gender, refeeding; inpatient, outcomes;

\section{Abbreviations}

AN-Anorexia Nervosa; ARFID-Avoidant/Restrictive Food Intake Disorder; BMI-Body Mass Index; BN-Bulimia Nervosa; DSM - Diagnostic and Statistical Manual of Mental Disorders; EBW-Expected Body Weight; EDNOS-Eating Disorders not otherwise Specified; IQR - Interquartile Range; LOS_Length Of Stay; OSFED-Other Specified Feeding and Eating Disorders

\section{Introduction}

Eating disorders have historically been portrayed as a female phenomenon. Recent estimates, however, suggest a growing prevalence of eating disorders among males, with males accounting for 10 to $25 \%$ of patients [1,2]. This percentage may further increase as clinicians adopt the latest changes to eating disorder criteria published in the Diagnostic and Statistical Manual of Mental Disorders, Fifth Edition (DSM-5) [3]. These changes, among others, include removing amenorrhea from the criteria for anorexia nervosa (AN) and adding diagnoses for binge eating disorder and Avoidant/Restrictive Food Intake Disorder (ARFID), which will likely add to the number of males with specified eating disorders $[4,5]$. Despite growing recognition of eating disorders in males, research rarely includes gender comparisons due to small sample sizes or exclusion of males, particularly among patients requiring hospitalization.

Restrictive eating disorders, such as AN and ARFID, often lead to a state of nutritional insufficiency, defined as inadequate nutrition to meet metabolic needs [6]. Treatment for nutritional insufficiency requires refeeding to replenish losses, restore function, and prevent deterioration throughout the body. This therapeutic process, however, can result in electrolyte shifts and subsequent organ dysfunction in a life-threatening condition called "refeeding syndrome" [7]. Hospitalization is therefore necessary in medically unstable patients to prevent potential consequences during initial refeeding. The optimal approach to refeeding safely as well as efficiently is a continued area of research without consensus and, of the studies available on refeeding protocols, [8]. none compare male and female responses to treatment.

Providers at the Cleveland Clinic have been using a clinical care path to treat both male and female patients hospitalized with nutritional insufficiency for over 20 years. This care path was adapted to an electronic medical records system beginning in 2002, with complete inpatient documentation available electronically since 2008 . The primary purpose of this study was 
to compare the success of the nutritional insufficiency care path at stabilizing and initiating recovery in male and female patients hospitalized between 2008 and 2014. Here we present gender differences identified at presentation, during hospitalization, and one year after admission. Recognizing these differences may help to both improve disease recognition and tailor treatments to better meet the needs of all eating disorder patients.

\section{Methods}

\section{Study Population and Design}

This study was approved by the Cleveland Clinic Institutional Review Board and informed consent was waived due to the retrospective design. We reviewed electronic medical records for patients aged 5 to 31 years hospitalized for the treatment of nutritional insufficiency between January 2008 and December 2014 at the Cleveland Clinic. Patients were included if they were treated using the nutritional insufficiency care path and excluded if they did not meet DSM-5 criteria for an eating disorder or were hospitalized for less than 24 hours. For patients readmitted during the study period, only the first admission was included for analysis.

Demographic information, eating disorder history, comorbid psychiatric diagnoses, and substance use were obtained from semi-structured interviews conducted by Adolescent Medicine specialists, when available, or other physicians involved in patient care. Eating disorder diagnoses were made according to DSM-5 criteria and confirmed by an Adolescent Medicine specialist [3]. Excessive alcohol use for patients under 21 years was considered any consistent use. Excessive alcohol use for patients over 21 years included binge drinking ( $>5$ drink in one sitting for males or $>4$ drinks in one sitting for females), heavy drinking ( $>14$ drinks per week in males or $>7$ drinks per week in females), or reporting a history of blackouts or pass-outs following alcohol use. Treatment variables, including length of stay (LOS), daily weights, daily caloric intake, daily vital signs, electrocardiogram findings, and the incidence of hypophosphatemia $(<2.5 \mathrm{mg} /$ $\mathrm{dL})$, hypomagnesemia $(<1.7 \mathrm{mg} / \mathrm{dL})$, and hypokalemia $(<3.5$ $\mathrm{mEq} / \mathrm{L}$ ), were recorded for each admission. Prolonged QTc was defined as greater than $450 \mathrm{~ms}$. Bradycardia was defined as less than $50 \mathrm{bpm}$ while awake or $40 \mathrm{bpm}$ while asleep. Hypotension was defined as a systolic blood pressure of less than $90 \mathrm{mmHg}$. Hypothermia was defined as less than $96^{\circ} \mathrm{F}$.

One year follow-up information was obtained for patients admitted prior to January 2014. Number of readmissions at the Cleveland Clinic within one year of initial admission was documented for each patient. Remission status was recorded for each patient using a physician or nurse note between 9 and 15 months of initial admission. Patient notes were obtained from either electronic records from the Cleveland Clinic or from the Emily Program, a local eating disorder treatment center. For patients without a follow-up within this timeframe, those who met the criteria for remission prior to 9 months were considered in remission and those who continued to exhibit disordered eating behaviors after 15 months were assumed to not be in remission at one year. Criteria for remission included $\geq 90 \%$ expected body weight (\%EBW) without evidence of weight loss behaviors, including restriction, excessive exercise, self-induced vomiting, and diuretic, laxative, or diet pill abuse, for at least three months. Excessive exercise was defined as $\geq 60$ minutes of exercise a day, on average, or regular exercise with moderate to severe distress when unable to exercise.

\section{Nutritional Insufficiency Care Path Protocol}

Patients were placed on bed rest with 24 hour supervision and continuous cardiac monitoring. Blinded weights were measured each morning with patients standing backwards on a digital scale in a single hospital gown after voiding. Height was measured once using a stadiometer during admission. Temperature and orthostatic vital signs were measured at least three times daily. Laboratory studies, including a complete metabolic panel, serum inorganic phosphorus, serum magnesium, complete blood count with differential, erythrocyte sedimentation rate, thyroid stimulating hormone, and urinalysis, were obtained within 24 hours of admission and as needed during admission. All patients received phosphorus supplementation (250 mg phosphorus, $164 \mathrm{mg}$ sodium, and $278 \mathrm{mg}$ potassium per packet) twice daily for 5 days. Other supplements were given as needed to correct laboratory abnormalities.

Dietitians met with patients within 24 hours of admission and prescribed initial calories based on patient presentation and diet history. Initial diets included a minimum of $1500 \mathrm{kcal} /$ day, with a majority of patients started on $2200 \mathrm{kcal} /$ day. Calories were increased during admission to meet a daily weight gain goal of about $0.2 \mathrm{~kg}$. Patients were required to finish their meals within 30 minutes and their snacks within 20 minutes. Unconsumed calories were replaced with a high-calorie supplement drink that, if refused orally, was given via nasogastric tube. Rare patients required regular nasogastric nutrition due to persistent food refusal.

Discharge criteria included resolution of bradycardia (> $50 \mathrm{bpm}$ while awake and $>40 \mathrm{bpm}$ while asleep), hypotension (systolic blood pressure $>90 \mathrm{mmHg}$ ), and hypothermia ( $>96^{\circ} \mathrm{F}$ ) for at least 24 hours and resolution of prolonged QTc $(\leq 450 \mathrm{~ms})$, electrolyte abnormities, and intractable vomiting. Patients with active suicidality required sufficient psychiatric follow-up. All patients and their families met with a physician trained in eating disorder treatment prior to discharge. During these conversations, the physician educated families on disordered eating and helped develop a post-hospitalization treatment plan. Most patients transitioned to outpatient treatment programs, typically familybased, at a local eating disorder treatment center.

\section{Data Analysis}

Rate of weight gain was determined by dividing the change in weight from the first full day of admission to the day of discharge by the number of days in between. Rate of caloric increase was determined by dividing the change in calories from the initial calorie prescription to the prescription on the last full day of admission (all three meals in hospital) by the number of days between consults. \%EBW was calculated by dividing the patient 
body mass index (BMI) by the median BMI for his or her age and multiplying by 100 . Median body mass index (BMI), the $50^{\text {th }}$ percentile BMI based on gender and age, was determined using the 2000 Centers for Disease Control and Prevention BMI-for-age growth charts for patients aged 2 to 20 years. For patients over 20 years, a median BMI of 21.8 and 23.04 was used for females and males, respectively.

Data were described using medians and Interquartile Ranges (IQR) for continuous variables and counts and percentages for categorical variables. Male and female patients were compared using the Kruskal-Wallis test for continuous variables and the Chi-square test or Fisher's exact test, if expected count was less than 5 in $20 \%$ of cells, for categorical variables. Differences in LOS were also assessed using the Kaplan-Meier method and Wilcoxon test. All analyses were two-tailed and performed at a significance level of 0.05. Results were analyzed using JMP 10.0 (SAS Institute Inc, Cary, NC).

A post-hoc multivariable analysis was performed to determine factors associated with LOS. Variables with significant univariate associations with LOS were entered into a multivariable linear regression model with stepwise selection of variables (entry $\mathrm{p}<$ 0.05 and exit $p>0.10$ with the F-test) $[13,14]$. Factors analyzed included gender, age, ethnicity, eating disorder diagnosis, duration of illness, weight loss, rate of weight loss, admission \%EBW, binge-eating, self-induced vomiting, weight loss substance use (i.e., laxative, diuretic, or diet pill use), excessive exercise, other psychiatric diagnosis, initial calories during hospitalization, initial calories per $\mathrm{kg}$, rate of caloric increase, primarily nasogastric nutrition ( $>50 \%$ of calories), primarily oral nutrition ( $>50 \%$ of calories), prolonged QTc, bradycardia, hypotension, hypothermia, abnormal admission electrolytes, and rate of weight gain.

\section{Results}

Between January 2008 and December 2014, 346 patients were placed on the nutritional insufficiency care path at the Cleveland Clinic. Five patients were excluded because they were hospitalized for less than 24 hours. One patient had nutritional insufficiency secondary to a metabolic disorder and therefore did not meet DSM-5 criteria for an eating disorder. The remaining patients included 38 males (11\%) and 302 females (89\%).

Patients included in the study were predominantly nonHispanic white $(95 \%, \mathrm{n}=324)$ with ages that ranged from 8 to 30 years (median age of 16 years in males and 17 years in females). Table 1 summarizes male and female disease characteristics at the time of initial admission. Most patients met DSM-5 criteria for anorexia nervosa $(66 \%, n=226)$, with no gender difference in eating disorder diagnoses. Female patients, however, were more likely to engage in certain disordered eating behaviors, including binge-eating, self-induced vomiting, and laxative, diuretic, or diet pill abuse. Male patients, in contrast, were more likely to use illicit drugs. Males consistently had shorter durations of illness with greater weight loss prior to admission, resulting in a faster rate of weight loss.
Gender comparisons during the course of hospitalization are summarized in Table 2. Most patients had severe bradycardia $(64 \%, \mathrm{n}=217)$ on admission, with many also experiencing hypotension $(51 \%, \mathrm{n}=175)$ and hypothermia ( $26 \%, \mathrm{n}=87)$. No gender differences in vital sign instability were identified. A higher percentage of female patients had electrocardiograms revealing prolonged QTc $(18 \%, \mathrm{n}=52)$ compared to male patients $(6 \%, \mathrm{n}$ $=2$ ), although the difference was not statistically significant ( $\mathrm{p}$ $=0.07$ ). Only one male patient was admitted with hypokalemia compared to 32 female patients $(\mathrm{p}=0.15)$. Four female patients were also admitted with hypophosphatemia and one male patient was admitted with hypomagnesemia. Other reasons for admission included intractable vomiting, hematemesis, severe anemia, altered mental status, and suicidality.

Both genders received similar dietary interventions during admission (Table 2), with two-thirds of patients prescribed at

Table 1: Gender comparison of eating disorder history at initial admission.

\begin{tabular}{|c|c|c|c|}
\hline & $\begin{array}{c}\text { Male } \\
\text { Patients }\end{array}$ & $\begin{array}{c}\text { Female } \\
\text { Patients }\end{array}$ & P Value \\
\hline & $(\mathrm{n}=38)$ & $(\mathrm{n}=302)$ & \\
\hline Age, median (IQR), y & $16(14-19)$ & $17(15-20)$ & 0.07 \\
\hline
\end{tabular}

Eating disorder diagnosis, \% (No.)

\begin{tabular}{|c|c|c|l|}
\hline Anorexia nervosa & $61(23)$ & $67(203)$ & 0.47 \\
\hline Bulimia nervosa & $3(1)$ & $7(22)$ & 0.49 \\
\hline ARFID & $16(6)$ & $12(36)$ & 0.44 \\
\hline Atypical anorexia nervosa & $21(8)$ & $13(39)$ & 0.21 \\
\hline Purging disorder & $0(0)$ & $1(2)$ & 1 \\
\hline
\end{tabular}

Eating disorder history, median (IQR)

\begin{tabular}{|c|c|c|c|}
\hline Illness duration, mo & $7(5-24)$ & $14(6-39)$ & 0.03 \\
\hline Weight loss, kg & $13(6-22)$ & $10(6-15)$ & 0.04 \\
\hline Rate of weight loss, kg/mo & $1.9(0.5-3.6)$ & $\begin{array}{c}0.8(0.3- \\
3.4)\end{array}$ & 0.004 \\
\hline Percent expected body weight, \%a & $86(76-97)$ & $85(78-96)$ & 0.69 \\
\hline
\end{tabular}

Eating disorder behaviors, \% (No.)

\begin{tabular}{|c|c|c|c|}
\hline Binge-eating & $13(5)$ & $29(88)$ & 0.04 \\
\hline Self-induced vomiting & $18(7)$ & $34(104)$ & 0.047 \\
\hline Weight loss substances $^{\mathrm{b}, \mathrm{c}}$ & $5(2)$ & $21(63)$ & 0.02 \\
\hline Excessive exercisec & $71(27)$ & $68(206)$ & 0.74 \\
\hline Comorbidity, \% (No.) & $32(12)$ & $41(122)$ & 0.29 \\
\hline Other psychiatric diagnosis $^{|l|}$ & $24(9)$ & $22(66)$ & 0.79 \\
\hline Excessive alcohol use $^{\mathrm{d}}$ & $21(8)$ & $5(16)$ & 0.002 \\
\hline Illicit drug use $^{\mathrm{d}}$ &
\end{tabular}

Abbreviation: ARFID, Avoidant/Restrictive Food Intake Disorder.

abased on median body mass index for age and gender of patient at time of admission.

bWeight loss substances include abuse of laxatives, diuretics, or diet pills.

cWeight loss substance and exercise data was unavailable for 1 female patient.

dAlcohol and drug use data was unavailable for 2 female patients. 
least 2000 calories during initial consultation and advanced daily by at least 250 calories. Rate of weight gain and \%EBW at discharge were consistent across genders. Despite similar interventions and weight gain, male patients were more likely to have longer hospitalizations than female patients ( 6.5 days vs. 5 days, $p=0.007$ ). Post-hoc multivariable analysis, however, revealed a non-significant relationship between LOS and gender when adjusted for other variables $(\mathrm{p}=0.08$, Table 3$)$. LOS on multivariable analysis was positively associated with duration of illness, hypothermia, nasogastric nutrition, slower weight gain, and slower caloric increase.

No clinical cases of refeeding syndrome occurred during admission. Two male patients and five female patients had documented electrolyte shifts during refeeding. Among female patients, shifts included hypophosphatemia $(n=2)$, hypokalemia $(\mathrm{n}=2)$, and hypomagnesemia $(\mathrm{n}=1)$. No male patients had documented hypophosphatemia during refeeding, but one male patient developed hypokalemia and another hypomagnesemia.

Table 2: Gender comparison during course of hospitalization.

\begin{tabular}{|c|c|c|c|}
\hline & $\begin{array}{c}\text { Male } \\
\text { Patients } \\
(n=38)\end{array}$ & $\begin{array}{l}\text { Female } \\
\text { Patients } \\
(n=302)\end{array}$ & $P$ Value \\
\hline \multicolumn{4}{|l|}{ Reasons for admission, \% (No.) } \\
\hline Bradycardia & $74(28)$ & 63 (189) & 0.18 \\
\hline Prolonged QTc ${ }^{\mathrm{a}}$ & $6(2)$ & $18(52)$ & 0.07 \\
\hline Electrolyte abnormalities $^{\mathrm{b}}$ & $5(2)$ & $12(35)$ & 0.28 \\
\hline \multicolumn{4}{|c|}{ Dietary intervention, median (IQR) } \\
\hline Initial calories, ${ }^{\mathrm{c}} \mathrm{kcal}$ & $\begin{array}{c}2300(1800- \\
2500)\end{array}$ & $\begin{array}{l}2200(1700- \\
2300)\end{array}$ & 0.06 \\
\hline $\begin{array}{c}\text { Initial calories per } \mathrm{kg},{ }^{\mathrm{c}} \mathrm{kcal} / \\
\mathrm{kg}\end{array}$ & $46(37-59)$ & $45(35-53)$ & 0.60 \\
\hline $\begin{array}{l}\text { Rate of caloric increase, } \\
\text { kcal/d }\end{array}$ & $\begin{array}{c}250(160- \\
410)\end{array}$ & $250(160-250)$ & 0.19 \\
\hline \multicolumn{4}{|c|}{ Route of caloric administration, \% (No.) } \\
\hline Any nasogastric nutrition & $13(5)$ & $9(26)$ & 0.36 \\
\hline $\begin{array}{c}\text { Primarily nasogastric } \\
\text { nutrition }^{\mathrm{e}}\end{array}$ & $11(4)$ & $5(16)$ & 0.20 \\
\hline Primarily oral nutrition ${ }^{\mathrm{e}}$ & $89(34)$ & $95(286)$ & 0.20 \\
\hline \multicolumn{4}{|c|}{ Treatment outcomes, median (IQR) } \\
\hline Length of stay, d & $6.5(4.7-10.0)$ & $5.0(3.0-7.0)$ & 0.007 \\
\hline Rate of weight gain, ${ }^{\mathrm{f}} \mathrm{kg} / \mathrm{d}$ & $0.4(0.2-0.5)$ & $0.3(0.2-0.5)$ & 0.53 \\
\hline $\begin{array}{l}\text { Percent expected body } \\
\text { weight, } \%\end{array}$ & $88(78-100)$ & 88 (81-98) & 0.91 \\
\hline
\end{tabular}

${ }^{\mathrm{a} E l e c t r o c a r d i o g r a m s}$ were unavailable for 15 females and 4 males. bElectrolyte abnormalities included hypokalemia ( 32 females, 1 male), hypophosphatemia ( 4 females), and hypomagnesemia ( 1 male).

Initial calorie data was unavailable for 3 females due to incomplete documentation.

${ }^{\mathrm{d} C}$ Caloric increase was unavailable for 38 females and 1 male patient due to short length of stay.

'Defined as greater than $50 \%$ of calories.

fWeight gain calculations did not include hospitalizations of $<2$ days (24 females, 2 males).

\begin{tabular}{|c|c|c|c|}
\hline \multirow[b]{2}{*}{ Variable } & \multirow{2}{*}{\begin{tabular}{|c|} 
Univariate \\
P Value
\end{tabular}} & \multicolumn{2}{|c|}{ Multivariable Model } \\
\hline & & $\begin{array}{c}\beta \\
\text { Coefficient }\end{array}$ & P Value \\
\hline \multicolumn{4}{|l|}{ Admission Variables } \\
\hline Male gender & 0.007 & 0.7447 & 0.08 \\
\hline ARFID diagnosis & 0.0004 & & \\
\hline Illness duration, mo & 0.002 & 0.0215 & 0.007 \\
\hline Weight loss, kg & 0.04 & & \\
\hline$\%$ Expected body weight & 0.005 & & \\
\hline \multicolumn{4}{|l|}{ Hospitalization Variables } \\
\hline Hypotension & 0.008 & & \\
\hline Hypothermia & 0.0001 & 1.4334 & $<0.0001$ \\
\hline Primarily nasogastric nutrition & $<0.0001$ & 3.7092 & $<0.0001$ \\
\hline Rate of caloric increase, $\mathrm{kcal} / \mathrm{d}$ & $<0.0001$ & -0.0057 & 0.0004 \\
\hline Rate of weight gain, $\mathrm{kg} / \mathrm{d}$ & 0.006 & -1.3922 & 0.04 \\
\hline
\end{tabular}

Abbreviations: ARFID, Avoidant/Restrictive Food Intake Disorder

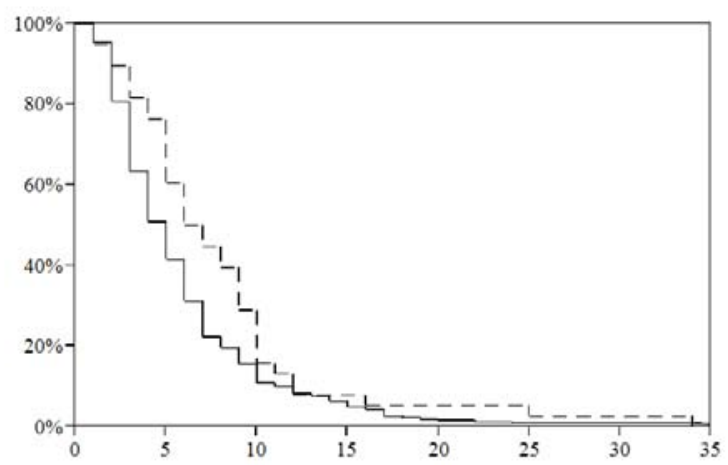

Figure 1:Percentage of patients remaining hospitalized

A majority of patients, however, did not have repeat laboratory studies during admission, including $65 \%(n=220)$ without repeat phosphorus levels, 68\% $(n=231)$ without repeat magnesium level, and $55 \%(n=186)$ without repeat potassium levels. There was no significant difference in the occurrence of electrolyte shifts or in the percentage of missing laboratory data among male and female patients.

One year follow-up information was obtained for patients admitted prior to January $2014(\mathrm{n}=304$, including 33 males and 271 females). Within one year of initial admission, 20\% of patients $(n=62)$ were readmitted, with no significant difference between male and female patients $(18 \%$ vs. $21 \%, p=0.74)$. Of these patients, 19 females (7\%) but no males were readmitted more than once throughout the year. Remission status was available for $67 \%$ of male patients $(\mathrm{n}=22)$ and $81 \%$ of female patients $(n=219)$. Of these patients, a higher percentage of male patients were recovered by one year than female patients, although the difference was not statistically significant (64\% vs. $49 \%, \mathrm{p}=0.19$ ). 


\section{Discussion}

This study presents gender differences among eating disorder patients hospitalized for medical stabilization. Prior to hospitalization, males experienced a faster weight loss despite reporting less purging behaviors than females. Males were also more likely to have a history of illicit drug use compared to females, with no difference in psychiatric comorbidity. During admission, although both genders received similar dietary interventions and experienced similar rates of weight gain, length of hospitalization was significantly longer for male patients. Long-term outcomes, however, were similar for males and females, with around half meeting criteria for remission and less than one-quarter requiring readmission within one year of hospitalization.

This study is one of the first to classify hospitalized patients using DSM-5 eating disorder criteria. In developing DSM-5, one of the primary goals was to modify classification to better describe patient behaviors and symptoms, therefore decreasing the number of patients given the DSM-IV diagnosis of Eating Disorders Not Otherwise Specified (EDNOS). [15]. In our patient population, a majority of patients met the updated criteria for AN (66\%) with less classified under Other Specified Feeding and Eating Disorders (OSFED, 14\% atypical AN and 0.6\% purging disorder), ARFID (12\%), or BN (7\%). These percentages align with those reported in outpatient populations using DSM-5 [16]. The percentage of OSFED patients, as expected, was lower than the percentage of EDNOS patients reported in previous studies using DSM-IV criteria [17]. Diagnoses did not differ significantly between genders. More research is needed to determine whether gender differences exist among patients with the same DSM-5 diagnosis.

Previous studies comparing male and female eating disorder phenotypes have reported variable results, with many identifying more similarities than differences. In our study population, males had shorter illnesses and faster weight loss than females prior to hospitalization. Two additional studies have reported shorter illnesses among males prior to presentation for inpatient or outpatient treatment $[18,19]$, although the gender difference was not statistically significant and neither study assessed rate of weight loss. Males in our study also participated in less disordered eating behaviors, other than excessive exercise, compared to females. This finding opposes a recent study on patients hospitalized with an that identified no significant gender difference in binge-eating or purging behaviors [18]. Studies that include a broader spectrum of eating disorders, however, have identified a lower prevalence of eating disorder behaviors among young men consistent with our findings [20, 22]. Excessive exercise is often distinguished from other behaviors and recognized as a common weight loss method in eating disorders [22, 23], particularly among males [24, 25]. In our study population, excessive exercise was prevalent in both males and females, highlighting this behavior as an important aspect of eating disorders regardless of gender.

Psychiatric conditions, including substance abuse, often occur concurrently with eating disorders in both males and females $[26,27]$. Many previous reports, however, have not compared comorbidity between male and female patients. In this study, we did not find a difference in comorbid psychiatric disorders among males and females. Male patients nevertheless were more likely than female patients to use illicit drugs. This finding may, in part, be due to the higher prevalence of illicit drug use among males in the general population [28]. More investigation is necessary to explore the potential relationship between drug use in males and severe nutritional insufficiency, which may impact treatment.

During hospitalization, male and female patients were refed using the same protocol with similar dietary interventions. LOS using this protocol was shorter than that typically reported in the literature [8, 29-31], with three-fourths of patients hospitalized for less than a week, regardless of gender. This finding adds to growing evidence that higher calorie diets promote weight gain and reduce LOS without increasing the risk of refeeding syndrome in patients with moderate malnutrition [30-32]. Reductions in LOS are advantageous not only because they reduce cost [33], but because they allow for earlier transition to family-based treatment in the outpatient setting, the most evidence-based treatment for restrictive eating disorders in adolescents [34-37].

Although the median LOS was low for both genders, male patients tended to be hospitalized for longer than female patients. Reasons for this difference were unclear given similar dietary interventions, rates of weight gain, and complications. Gender was not significantly associated with LOS ( $p=0.08$ ), however, when adjusted for other variables in a post-hoc multivariable analysis. Significant predictors of longer LOS included longer duration of illness prior to admission and hypothermia, primarily nasogastric nutrition, slower caloric increase, and slower weight gain during admission. These results highlight the importance of optimizing the dietary intervention to efficiently stabilize both male and female patients.

One year after hospitalization, half of our study population achieved remission, defined as $\geq 90 \%$ EBW and absence of eating disorder behaviors for at least three months. This finding is consistent with remission rates for other programs. In a multicenter study of eating disorder programs across the nation, for example, $40 \%$ to $63 \%$ of patients achieved weight restoration ( $\geq 90 \%$ EBW) within one year of treatment. The percentage of male patients in remission in our study was higher than that of female patients (64\% vs. $49 \%$ ), although the difference was not statistically significant. In one other study comparing remission rates in male and female patients enrolled in an eating disorder program, the median time to remission was significantly lower in males than in females ( 3 years vs. 7 years), although the authors did not report on remission at one year. Readmission rates in our population were also similar to those reported in the literature [29]. Interestingly, although overall readmission did not differ across gender, no male patients required more than one readmission within a year, compared to $7 \%$ of females.

We recognize several limitations in this study. First, data were collected retrospectively from medical records, inherently 
limiting the information available for analysis. The reported number of electrolyte shifts during refeeding, for example, may be underestimated since a majority patients did not have repeat laboratory studies recorded during refeeding. In addition, the absence of data on treatment following discharge, readmission to other institutions, and remission for $21 \%$ of patients $(n=63)$ limits our ability to interpret follow-up data. Lastly, the study population consisted of predominantly white patients with moderate malnutrition. Our results therefore do not necessarily apply to other groups of patients, including other ethnicities and patients with severe malnutrition.

In conclusion, this study identifies multiple gender differences among patients hospitalized with nutritional insufficiency, including variations in course of illness and course of hospitalization. While eating disorder patients share many similarities across gender, their differences may affect their needs both during and after hospitalization. Despite these differences, however, male and female patients had similar outcomes one year after hospitalization, highlighting both the success and continued need for improvement in eating disorder treatment following hospitalization in male and female patients.

\section{References}

1. Rosen DS. American Academy of Pediatrics Committee on Adolescence. Identification and management of eating disorders in children and adolescents. Pediatrics. 2010;126(6):1240-1253. doi:10.1542/ peds.2010-2821.

2. Sigel E. Eating disorders. Adolesc Med State Art Rev. 2008;19(3):547572. xi.

3. American Psychiatric Association. Diagnostic and Statistical Manual of Mental Disorders, Fifth Edition. Washington, DC; 2013.

4. Fisher MM, Rosen DS, Ornstein RM, Mammel KA, Katzman DK, Rome ES, et al. Characteristics of avoidant/restrictive food intake disorder in children and adolescents: a "new disorder" in DSM-5. J Adolesc Health. 2014;55(1):49-52. doi:10.1016/j.jadohealth.2013.11.013.

5. Smink FRE, van Hoeken D, Oldehinkel AJ, Hoek HW. Prevalence and severity of DSM-5 eating disorders in a community cohort of adolescents. Int J Eat Disord. 2014;47(6):610-619. doi:10.1002/ eat. 22316.

6. Auron M, Rome E. Anorexia nervosa and bulimia nervosa: what the hospitalist needs to know about CPT 269.9, or nutritional insufficiency. Am Coll Physicians Hosp. 2011:38-45.

7. Mehanna HM, Moledina J, Travis J. Refeeding syndrome: what it is, and how to prevent and treat it. BMJ. 2008;336(7659):1495-1498. doi:10.1136/bmj.a301.

8. Garber AK, Michihata N, Hetnal K, Shafer M-A, Moscicki A-B. A prospective examination of weight gain in hospitalized adolescents with anorexia nervosa on a recommended refeeding protocol. J Adolesc Health Off Publ Soc Adolesc Med. 2012;50(1):24-29. doi:10.1016/j.jadohealth.2011.06.011.

9. Lock J. How clinical pathways can be useful: an example of a clinical pathway for the treatment of anorexia nervosa in adolescents. Clin Child Psychol Psychiatry. 1999;4(3):331-340. doi:10.1177/1359104 599004003004.

10. Sylvester CJ, Forman SF. Clinical practice guidelines for treating restrictive eating disorder patients during medical hospitalization.
Curr Opin Pediatr. 2008;20(4):390-397. doi:10.1097/ MOP.0b013e32830504ae.

11. Leclerc A, Turrini T, Sherwood K, Katzman DK. Evaluation of a nutrition rehabilitation protocol in hospitalized adolescents with restrictive eating disorders. J Adolesc Health Off Publ Soc Adolesc Med. 2013;53(5):585-589. doi:10.1016/j.jadohealth.2013.06.001.

12. Rocks T, Pelly F, Wilkinson P. Nutrition therapy during initiation of refeeding in underweight children and adolescent inpatients with anorexia nervosa: a systematic review of the evidence. J Acad Nutr Diet. 2014;114(6):897-907. doi:10.1016/j.jand.2013.11.022.

13. Ackard DM, Richter S, Egan A, Cronemeyer C. Poor outcome and death among youth, young adults, and midlife adults with eating disorders: an investigation of risk factors by age at assessment. Int J Eat Disord. 2014;47(7):825-835. doi:10.1002/eat.22346.

14. Bursac Z, Gauss CH, Williams DK, Hosmer DW. Purposeful selection of variables in logistic regression. Source Code Biol Med. 2008;3:17. doi: 10.1186/1751-0473-3-17.

15. Call C, Walsh BT, Attia E. From DSM-IV to DSM-5: changes to eating disorder diagnoses. Curr Opin Psychiatry. 2013;26(6):532-536. doi:10.1097/YCO.0b013e328365a321.

16. Ornstein RM, Rosen DS, Mammel KA, Callahan ST, Forman S, Jay MS, et al. Distribution of eating disorders in children and adolescents using the proposed DSM-5 criteria for feeding and eating disorders. J Adolesc Health Off Publ Soc Adolesc Med. 2013;53(2):303-305. doi:10.1016/j.jadohealth.2013.03.025.

17. Dalle Grave R, Calugi S. Eating disorder not otherwise specified in an inpatient unit: the impact of altering the DSM-IV criteria for anorexia and bulimia nervosa. Eur Eat Disord Rev J Eat Disord Assoc. 2007;15(5):340-349. doi:10.1002/erv.805.

18. Gueguen J, Godart N, Chambry J, Brun-Eberentz A, Foulon C, Divac Phd $\mathrm{SM}$, et al. Severe anorexia nervosa in men: comparison with severe AN in women and analysis of mortality. Int J Eat Disord. 2012;45(4):537545. doi:10.1002/eat.20987.

19. Støving RK, Andries A, Brixen K, Bilenberg N, Hørder K. Gender differences in outcome of eating disorders: a retrospective cohort study. Psychiatry Res. 2011;186(2-3):362-366. doi:10.1016/j. psychres.2010.08.005.

20. Mond J, Hall A, Bentley C, Harrison C, Gratwick-Sarll K, Lewis V. Eating-disordered behavior in adolescent boys: eating disorder examination questionnaire norms. Int J Eat Disord. 2014;47(4):335341. doi:10.1002/eat.22237.

21. Button E, Aldridge S, Palmer R. Males assessed by a specialized adult eating disorders service: patterns over time and comparisons with females. Int J Eat Disord. 2008;41(8):758-761. doi:10.1002/eat.20553.

22. Lewinsohn PM, Seeley JR, Moerk KC, Striegel-Moore RH. Gender differences in eating disorder symptoms in young adults. Int J Eat Disord. 2002;32(4):426-440. doi:10.1002/eat.10103.

23. Sundgot-Borgen J, Torstveit MK. Prevalence of eating disorders in elite athletes is higher than in the general population. Clin J Sport Med. 2004;14(1):25-32.

24. Hausenblas HA, Downs DS. Relationship among sex, imagery, and exercise dependence symptoms. Psychol Addict Behav J Soc Psychol Addict Behav. 2002;16(2):169-172.

25. Holland LA, Brown TA, Keel PK. Defining features of unhealthy exercise associated with disordered eating and eating disorder diagnoses. Psychol Sport Exerc. 2014;15(1). doi:10.1016/j. psychsport.2013.10.005. 
26. Bramon-Bosch E, Troop NA, Treasure JL. Eating disorders in males: a comparison with female patients. Eur Eat Disord Rev. 2000;8(4):321-328. doi:10.1002/1099-0968(200008)8:4<321::AIDERV355>3.0.CO;2-G.

27. Carlat DJ, Camargo CA, Herzog DB. Eating disorders in males: a report on 135 patients. Am J Psychiatry. 1997;154(8):1127-1132.

28. Swendsen J, Burstein M, Case B, Conway KP, Dierker L, He J, et al. Use and abuse of alcohol and illicit drugs in us adolescents: results of the national comorbidity survey-adolescent supplement. Arch Gen Psychiatry. 2012;69(4):390-398. doi:10.1001/ archgenpsychiatry.2011.1503.

29. Wiseman CV, Sunday SR, Klapper F, Harris WA, Halmi KA. Changing patterns of hospitalization in eating disorder patients. Int J Eat Disord. 2001;30(1):69-74. doi:10.1002/eat.1055.

30. Garber AK, Mauldin K, Michihata N, Buckelew SM, Shafer M-A, Moscicki A-B. Higher calorie diets increase rate of weight gain and shorten hospital stay in hospitalized adolescents with anorexia nervosa. J Adolesc Health. 2013;53(5):579-584. doi:10.1016/j. jadohealth.2013.07.014.

31. Golden NH, Keane-Miller C, Sainani KL, Kapphahn CJ. J Adolesc Health Off Publ Soc Adolesc Med. 2013;53(5):573-578. doi:10.1016/j. jadohealth.2013.05.014.
32. Whitelaw M, Gilbertson H, Lam P-Y, Sawyer SM. Does aggressive refeeding in hospitalized adolescents with anorexia nervosa result in increased hypophosphatemia? J Adolesc Health. 2010;46(6):577-582. doi:10.1016/j.jadohealth.2009.11.207.

33. Toulany A, Wong M, Katzman DK, Nadia Akseer, Steinegger, Rebecca L. Hancock-Howard, et al. Cost analysis of inpatient treatment of anorexia nervosa in adolescents: hospital and caregiver perspectives. CMAJ Open. 2015;3(2):E192-E197. doi:10.9778/cmajo.20140086.

34. Lock J, Le Grange D, Agras WS, Moye A, Bryson SW, Jo B. Randomized clinical trial comparing family-based treatment with adolescentfocused individual therapy for adolescents with anorexia nervosa. Arch Gen Psychiatry. 2010;67(10):1025-1032. doi:10.1001/ archgenpsychiatry.2010.128.

35. Eisler I, Simic M, Russell GFM, Dare C. A randomised controlled treatment trial of two forms of family therapy in adolescent anorexia nervosa: a five-year follow-up. J Child Psychol Psychiatry. 2007;48(6):552-560. doi:10.1111/j.1469-7610.2007.01726.x.

36. Le Grange D. The Maudsley family-based treatment for adolescent anorexia nervosa. World Psychiatry. 2005;4(3):142-146.

37. Robin AL, Siegel PT, Moye AW, Gilroy M, Dennis AB, Sikand A. A controlled comparison of family versus individual therapy for adolescents with anorexia nervosa. J Am Acad Child Adolesc Psychiatry. 1999;38(12):1482-1489. doi:10.1097/00004583-199912000-00008. 